\title{
Tick-Borne Encephalitis Virus Infection of Cultured Mouse Macrophages
}

\author{
Arunee Ahantarig $^{\mathrm{a}} \quad$ Daniel Růžek $^{\mathrm{b}}$ Marie Vancováb Anna Janowitz ${ }^{c}$ \\ Hana Štastnáb Martina Tesařováb Libor Grubhoffer ${ }^{b}$ \\ a Department of Biology, Faculty of Science, Mahidol University, Bangkok, Thailand; 'b Institute of Parasitology, \\ Biology Centre of the Academy of Sciences of the Czech Republic and Faculty of Science, University of South \\ Bohemia, České Budějovice, Czech Republic; ' Division of Infection and Immunity, Faculty of Biological and Life \\ Sciences, University of Glasgow, Glasgow, UK
}

\section{Key Words}

Tick-borne encephalitis virus · Scanning electron microscopy • Transmission electron microscopy • Macrophages $\cdot$ Virulence $\cdot$ Flavivirus

\begin{abstract}
The interactions of tick-borne encephalitis virus (TBEV) with mouse macrophages were studied at the electron microscopic level. The cultured mouse macrophages were sensitive to infection with TBEV strain Hypr (a highly neuroinvasive and neurovirulent strain for laboratory mice) and produced relatively high virus titers. However, these macrophage cells remained morphologically inactivated. Viral particles were located mainly in the ER but were also present in other exocytic compartments. No virus production was observed in cells infected with the attenuated, non-neuroinvasive TBEV strain 263. In this case, the infection led to a clear morphological activation of the macrophages. In conclusion, the virus replication process in mouse macrophage cells might be different from that in other mammalian cell lines since the smooth membrane structures, which are thought to be the sites for flavivirus replication, were not observed. Moreover, different TBEV strains exhibited a different interaction with the host macrophages. The inability
\end{abstract}

\section{KARGER}

Fax +41613061234 E-Mail karger@karger.ch www.karger.com
(C) 2009 S. Karger AG, Basel

$0300-5526 / 09 / 0525-0283 \$ 26.00 / 0$

Accessible online at:

www.karger.com/int of strain 263 to replicate in mouse macrophages as the first site of significant viral replication in vivo could be associated with the inability of this strain to establish a serious infection in mice.

Copyright $\odot 2009$ S. Karger AG, Basel

Tick-borne encephalitis (TBE) is a serious neuroinfection of viral etiology prevalent in wide areas of Europe and north-eastern Asia. Activation of macrophages and modulation of their effector functions is believed to be an integral part of TBE pathogenesis. The interaction between the TBE virus (TBEV; Hypr strain) and mouse peritoneal macrophages was studied previously [1]. Up until now, there have been several ultrastructural studies done on the TBEV that have shown that the TBEV maturation pathway in mammalian host cell lines differs from the pathway that the virus follows in tick vector cell lines [2], but no ultrastructural work of the effects on the TBEV strains on cultured mouse macrophages has been reported. Individual organism pathogenesis or other medical

\section{A.A. and D.R. contributed equally to the work.}

Dr. Arunee Ahantarig

Department of Biology

Faculty of Science, Mahidol University

Rama 6 Road, Bangkok 10400 (Thailand)

Tel. +662 201 5380, Fax +662 354 7161, E-Mail scaah@mahidol.ac.th 
issues related to the TBEV are usually studied in mouse models; therefore, mouse macrophage cell line could help to reveal what occurs in mice soon after infection [3-5]. Cells of the monocyte/macrophage lineage were recently demonstrated to represent an important source of local virus replication before viremia occurs [6]. Thus, in this work, transmission electron microscopy (TEM) was used for the first time to demonstrate ultrastructural changes of mouse macrophage infected with TBEV. Two low-passage members of the European TBEV subtype, a strain highly neuroinvasive and neurovirulent for laboratory mice (Hypr) [7], and a strain with a nonvirulent temperature-sensitive phenotype (263) [8] were used in this study.

The cell line of mouse macrophages IC-21 [9] was cultured in RPMI 1640 medium (Sigma) at $37^{\circ}$, supplemented by $10 \%$ fetal calf serum.

Cells grown in 96 -well plates $\left(1 \times 10^{5}\right.$ cells per well $)$ were inoculated with the virus (strain Hypr or 263) diluted in a small volume of culture medium, giving the multiplicity of infection 0.1 plaque-forming units (PFU)/ cell). After $1 \mathrm{~h}$ at $37^{\circ}$, free virus was removed by serial washing with phosphate-buffered saline (PBS) and, finally, $0.2 \mathrm{ml}$ of medium was added to each well. Virusmediated cytopathic effect was examined using light microscopy. The supernatant medium from appropriate wells was collected and frozen at $-70^{\circ}$ at $24,48,72,96$ and $120 \mathrm{~h}$ postinfection time periods. The virus titers were determined by plaque assay on porcine kidney stable (PS) cell monolayers, as described previously [10]. Briefly, tenfold dilutions of virus were placed in 24-well tissue culture plates to which a suspension of PS cells was added $\left(0.6-1.5 \times 10^{5}\right.$ cells per well). After an incubation for $4 \mathrm{~h}$, the suspension was overlaid with carboxymethylcellulose (1.5\% in L-15 medium). After incubating at $37^{\circ}$ and $0.5 \%$ $\mathrm{CO}_{2}$ for 5-6 days, the infected plates were washed with saline, and cell monolayers were stained with naphthalene black. Infectivity was expressed as PFU/ml.

For scanning electron microscopy, the culture medium from the infected macrophage culture (24-hour infection: Hypr and 263 strains) and uninfected cells was replaced with $2.5 \%$ glutaraldehyde in $0.1 \mathrm{M}$ phosphate buffer $(\mathrm{PB})$ and fixed overnight at $4^{\circ}$. The cells were rinsed three times with $0.1 \mathrm{M} \mathrm{PB}$ with $4 \%$ glucose (washing buffer), and postfixed for $1 \mathrm{~h}$ in $1 \%$ osmium tetroxide. After washing, the cells were dehydrated in increasing concentrations of acetone, followed by a drying step in Pelco CPD, and finally coated with gold. The photographs were obtained using a field emission JEOL JSM 7401-F SEM.

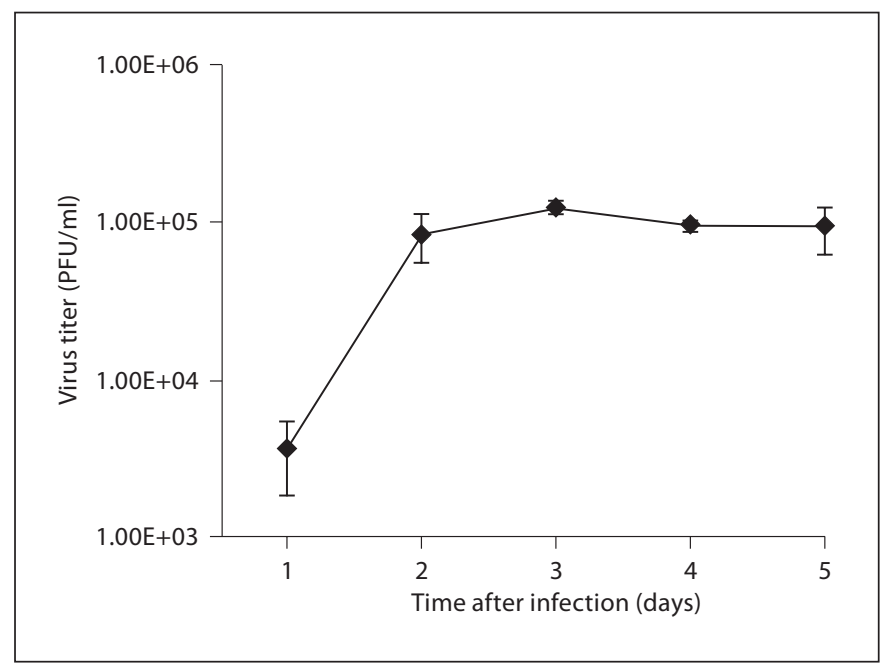

Fig. 1. Growth of the high-virulence TBEV strain (Hypr) in cultured mouse macrophages. The viral titers were quite stable after 2 -day infection. In the case of the low-virulence strain (263), the virus was detected only at $24 \mathrm{~h}$ after infection $\left(4.63 \times 10^{2} \pm 97.4\right.$ $\mathrm{PFU} / \mathrm{ml}$ ); at later time point intervals, the virus titer was under the detection limit.

When preparing the samples for TEM, the culture medium from the infected and uninfected macrophage culture was replaced with a fixation solution of $2.5 \%$ glutaraldehyde in $0.1 \mathrm{M} \mathrm{PB}$, and fixed overnight at $4^{\circ}$. The cells were rinsed three times with a washing buffer, scraped off from the glass slides, and embedded in $2 \%$ agar. Washed and dehydrated specimens were infiltrated and finally embedded in the SPI-Pon 812 resin. Ultrathin sections were cut using an Ultracut UCT microtom (Leica), counterstained with uranyl acetate and lead citrate, and finally examined in a JEOL JEM-1010 electron microscope at an acceleration voltage of $80 \mathrm{kV}$, equipped with a MegaView III camera (SIS GmbH).

In the case of immunofluorescence experiments, the culture medium from the infected culture was replaced with ice-cold methanol at $-20^{\circ}$ for $15 \mathrm{~min}$. The cells were washed three times, 10 min each with PBS, permeabilized with $1 \%$ Triton X-100 in PBS for 15 min. Cells on the glass slides were blocked with $0.05 \%$ Tween $20,10 \%$ normal goat serum and $0.002 \%$ BSA in PBS. All subsequent incubation and washing steps were carried out with PBS buffer. Double labeling was performed for $16 \mathrm{~h}$ at $4^{\circ}$ using anti-E antibody (1:200) and $\alpha$-tubulin $(1 \mu \mathrm{g} / \mathrm{ml})$ or $\gamma$-tubulin $(0.3 \mu \mathrm{g} / \mathrm{ml})$ antibodies. After washing, the cells were incubated in the dark at room temperature for 30 min with secondary antibodies conjugated with FITC or 
Fig. 2. Morphological changes on the surfaces of Hypr- and 263-infected mouse macrophages visualized using scanning electron microscopy. a Macrophages incubated without viruses have round shapes with smooth surfaces. b Cultured macrophages infected with TBE viruses (Hypr) have rugged surface with lamellipodia (black arrowhead and white arrow) and necrotic cell debris (black arrow). c Higher magnification of the control macrophage showed the presence of filopodia (white arrowhead) and cell surface ruffles (asterisk). d Infected macrophages exhibit numerous very long filopodia (arrow). The cell surface was covered with cell remnants. e 263-infected macrophage. Bars = $1 \mu \mathrm{m}$.
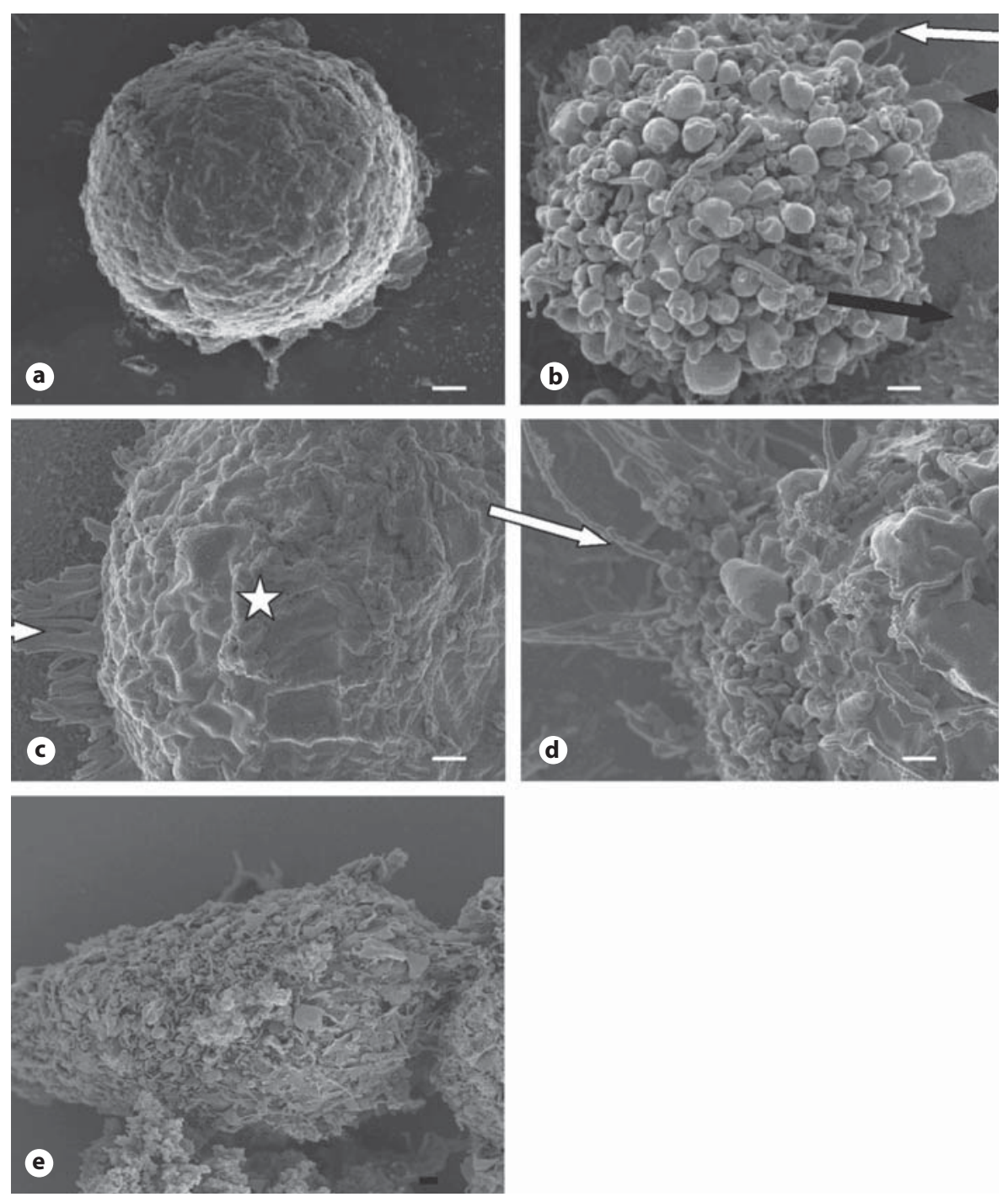

Cy3. The cells were washed five times, stained with DAPI $(1 \mu \mathrm{g} / \mathrm{ml})$ and after another washing step mounted with DABCO antifading agent (Sigma). All images were collected by a Zeiss fluorescent microscope.

The growth kinetics of the TBEV strains 263 and Hypr in IC-21 were studied. The cells were infected with multiplicity of infection 0.1 and the virus titers in the cultured medium were determined by plaque assay. The Hypr strain replicated relatively efficiently in the macrophage cells, reaching a titer of $10^{5} \mathrm{PFU} / \mathrm{ml}$ (fig. 1). Despite efficient virus production, the cells exhibited only minor morphological signs of activation as the macrophage morphologies were still round. In contrast, macrophage infection with the 263 strain was not productive (although the accumulations of the antigen in the cytoplasm of the infected cells were seen; see fig. 3), but the infection was associated with clear morphological activation of the macrophages, which exhibited increased cell surface ruffling and cell flattening around the macrophage surface (fig. 2e).

Mouse macrophage cells infected with the Hypr strain showed numerous lamellopodia (fig. 2b: white arrow) on the mobile edge of the cells including longer filopodia (fig. 2d: white arrow) compared with the smooth surface of the control macrophage (fig. 2c). Furthermore, the surfaces of infected macrophages were surrounded with necrotic cell debris (fig. 2b: black arrow). In contrast, macrophage cells infected with the strain 263 demonstrated morphological activation of macrophages with elongated shape (fig. 2e). 
Fig. 3. Ultrastructural features of Hyprand 263-infected cultured macrophages. a Uninfected cell. b Viruses (Hypr strain) presented on plasma membrane (arrowhead) and inside of phagosomes (arrow) that were in close proximity with the lysosome (LY). c Hypr strain assembled inside of the cell led to disorganization and proliferation of RER surrounded by mitochondria. d Higher magnification of c. The presence of Hypr viruses (arrows) in cisternae of RER is shown. e Necrotic Hypr-infected cell. $\mathbf{f}$ Engulfing of necrotic cell debris by other macrophages (Hypr infected). g Observation of ultrastructural changes of 263-infected macrophage cells. Numerous vesicles (V) containing viruslike particles and LY were observed in the cytoplasm. h High magnification of a 263infected macrophage showing vacuoles and fragmented ER. Bars $=1(\mathbf{a}, \mathbf{b}, \mathbf{d}-\mathbf{f}, \mathbf{h})$ and $2 \mu \mathrm{m}(\mathbf{c}, \mathbf{g})$.
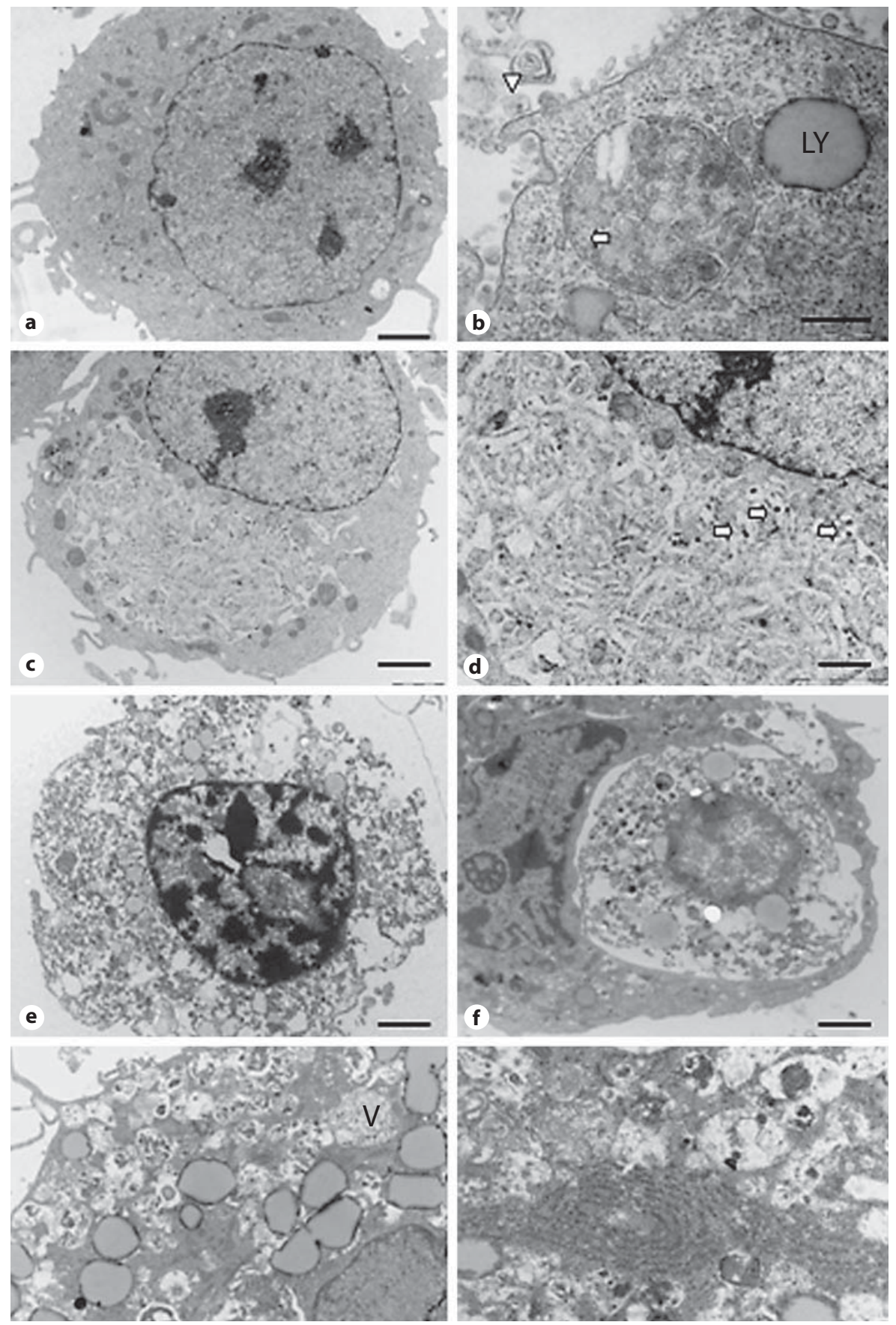

The cytoplasm of macrophage cells infected with the Hypr strain contained disorganized and proliferated cisternae of rough endoplasmic reticulum (RER) that were surrounded by numerous mitochondria (fig. 3c). Infected macrophages contained lysosomes (fig. $3 \mathrm{e}, \mathrm{f}$ ) and debris of necrotic cells that accommodated numerous viral particles. Viruses were engulfed alone or together with cel- lular debris during phagocytosis (fig. 3f). Virus particles were most frequently observed inside the lumen of the ER (fig. 3c, d). Furthermore, the infected cells contained virus particles in the lumen of the various compartments of the secretory pathway (data not shown). In addition, necrotic macrophages predominated over apoptotic ones. Morphological features of necrotic death were plasma 
E protein
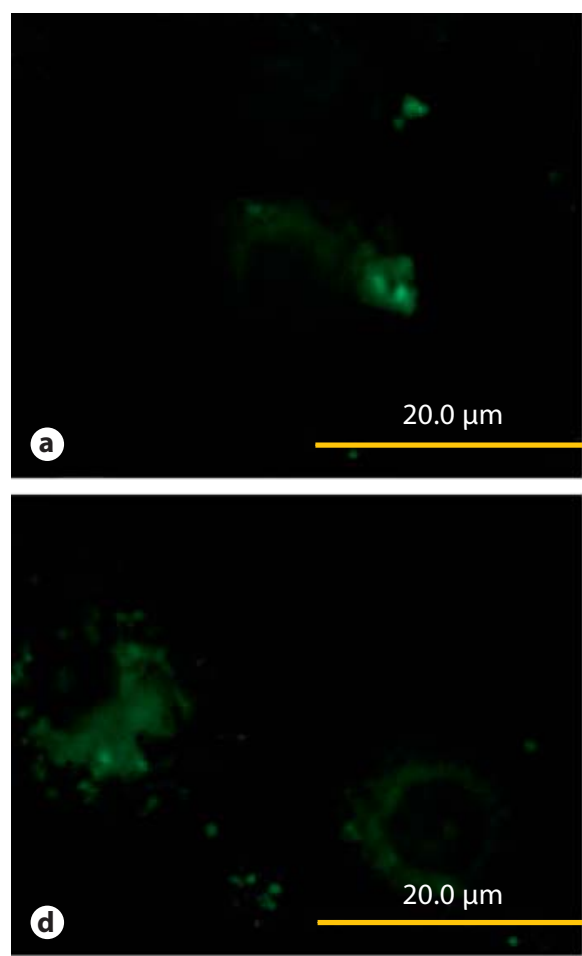

$\alpha$-Tubulin
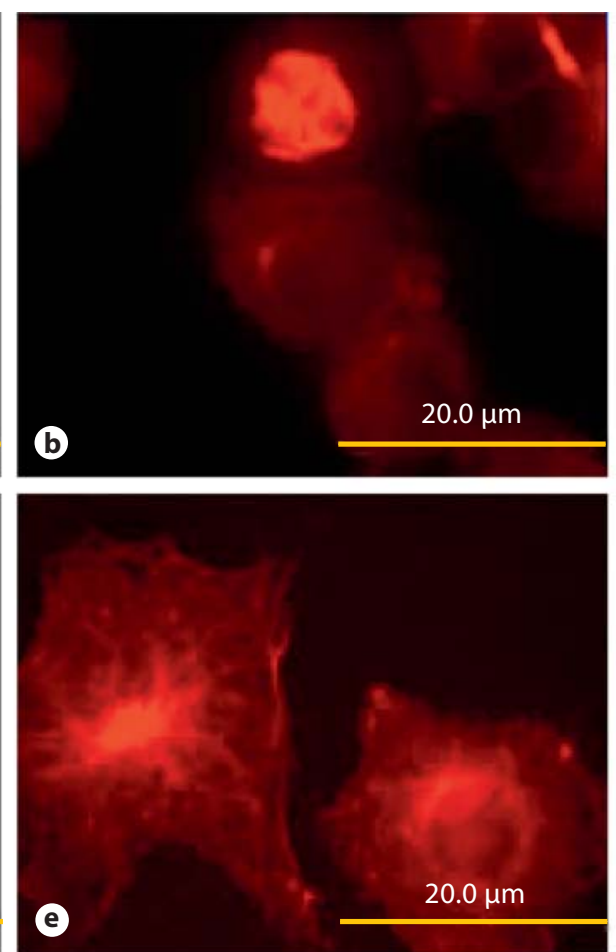

Merge and DAPI
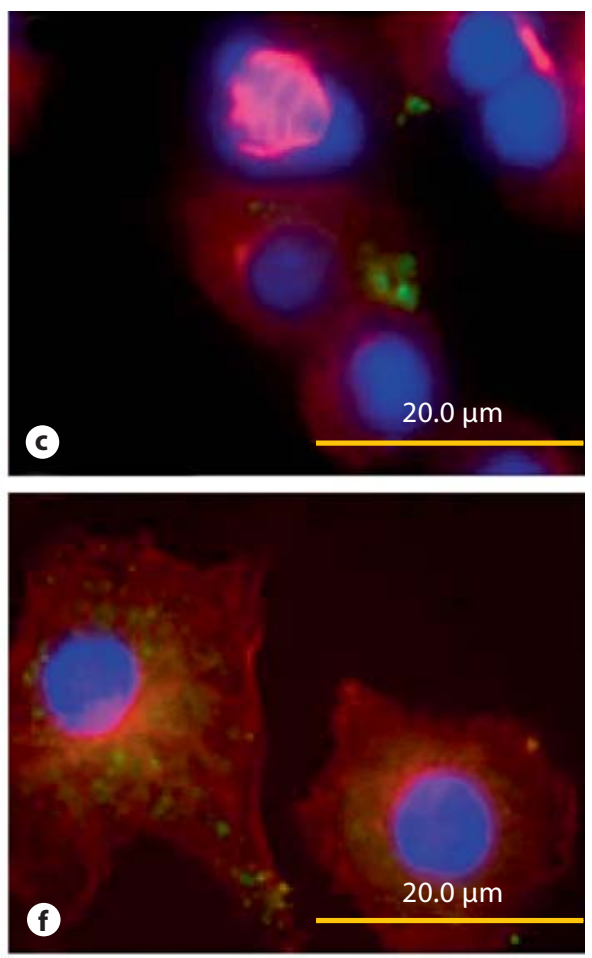

Fig. 4. Localization of TBE virus E protein and $\alpha$-tubulin protein. Mouse macrophage cells infected with Hypr and 263 strains were fixed and subjected to immunofluorescence staining with primary antibodies that recognize TBEV E protein and antibody against a marker for $\alpha$-tubulin protein. Bars $=20 \mu \mathrm{m}$. a-c Hypr-infected macrophages. d-f 263-infected macrophage cells. g Control (uninfected).

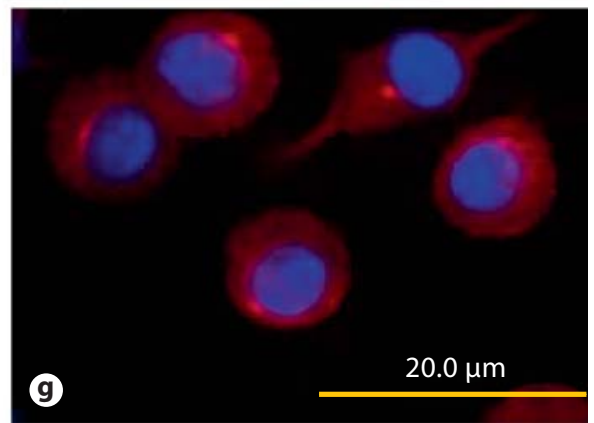

membrane disruption/disintegration and organelle swelling. The nucleus was still intact and chromatin distribution was regular (fig. 3e). No morphological changes were observed in the uninfected macrophage (control; fig. 3a).

No virus replication was observed in macrophages infected with the nonvirulent temperature-sensitive strain 263. Nevertheless, numerous vesicles containing viruslike particles, lysosomes and fragmented ER were observed in these macrophage cells (fig. 3g, h). Most organelles in the cytoplasm of macrophages were chaotic. Disruption of ER was seen (fig. 3h) and lysosome numbers were higher than usual (fig. $3 \mathrm{~g}$, h ) when compared to the uninfected macrophage cells (fig. 3a).

Tick-Borne Encephalitis Virus Infection of Cultured Mouse Macrophages
It was very difficult to observe microtubular localization or alterations from the ultrastructural images; therefore, immunofluorescence stainings of $\alpha / \gamma$-tubulin were done to determine the organization of these structures. Mouse macrophage cells were infected with either the Hypr or 263 strains (separate experiments). Visualization of the intracellular distribution of TBEV E protein, along with $\alpha / \gamma$-tubulin (either one), was performed. The cells were double stained using antibody against either anti- $\alpha$ or anti- $\gamma$-tubulin and with viral envelope protein E. Positive viral surface E protein staining was typically localized at the perinuclear region and in the cytoplasm of the Hypr- and 263-infected macrophage cells (fig. 4a, c, d, f and fig. $5 \mathrm{a}, \mathrm{c}, \mathrm{d}, \mathrm{f})$. No alteration of $\gamma$-tubulin was re-

Intervirology 2009;52:283-290 

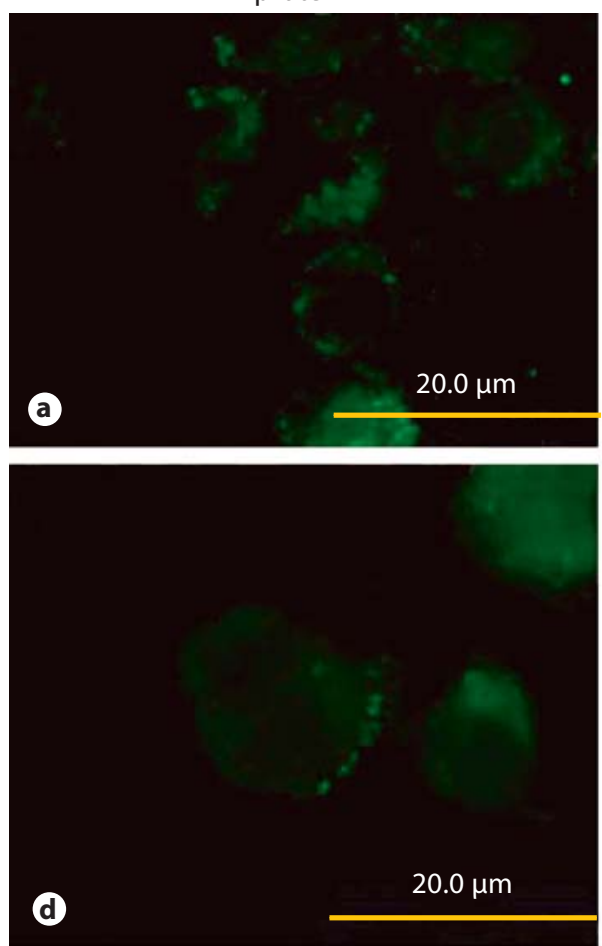

$\gamma$-Tubulin
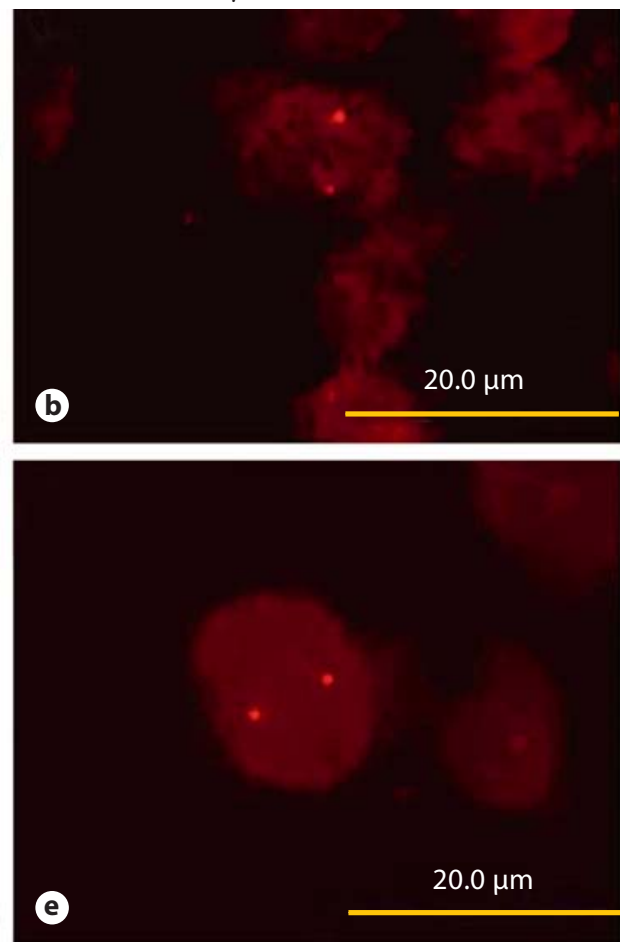
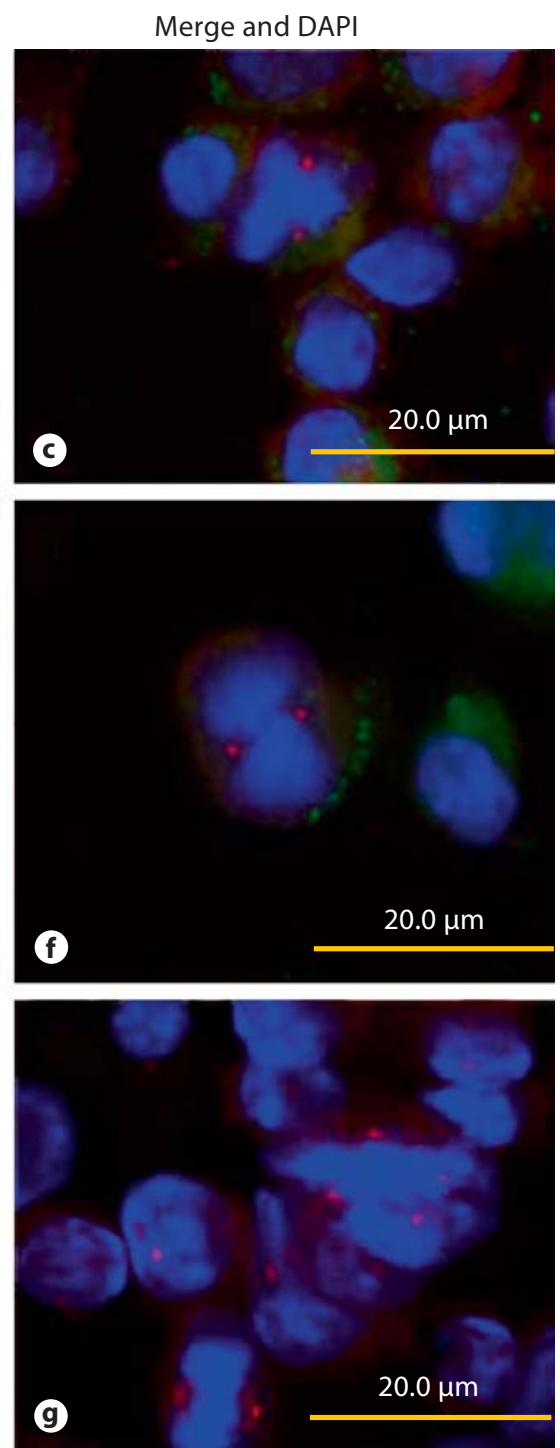

Fig. 5. Intracellular localization of TBEV E protein and $\gamma$-tubulin protein. Mouse macrophage cells infected with Hypr and 263 strains were fixed and subjected to immunofluorescence staining with primary antibodies that recognize TBEV E protein and antibody against a marker for $\gamma$-tubulin protein. Bars $=20 \mu \mathrm{m}$. a-c Hypr-infected macrophages. $\mathbf{d}-\mathbf{f}$ 263-infected macrophage cells. g Control (uninfected).

vealed in macrophages infected with either the Hypr or 263 strain (fig. 5g; control); and infected cells (fig. 5b, e). In contrast, cytoskeleton components, like the microtubules ( $\alpha$-tubulin), showed numerous alterations ( $\mathrm{Hypr}$ infected: fig. 4b) compared to the uninfected cells (fig. 4g). The $\alpha$-tubulin labeling of the cytoskeleton showed that the cells infected with the Hypr virus were rounded and microtubules were disorganized (fig. $4 \mathrm{~b}, \mathrm{c}$ ) when compared to uninfected control macrophages (fig. 4g). However, no disruptions of microtubule networks for $\alpha$-tubulin patterns were observed for the 263-infected macrophages (fig. 4e).

Generally, the assembly site of flaviviruses is ER, and the replication sites are on the cytoplasmic membranes
[11]. Formation of smooth membrane structure, which is thought to be a site of flavivirus replication [12], was observed in TBEV-infected glioblastoma and medulloblastoma cells, but not neuroblastoma cells [13] and was identified also in Kujin virus-infected Vero cells [14]. However, we revealed that smooth membrane structures were not formed in TBEV-infected macrophages. This might indicate that the virus replication process in mouse macrophage cells might be different from other non-macrophage cell types.

TBEV particles were located mainly in the ER but were also present in other exocytic compartments. In addition, the Hypr viral replication caused the proliferation and disorganization of the ER. The site of replication was sur- 
rounded by numerous mitochondria. The results are in accordance with observations of the infection of TBEV in neuronal cell cultures [13] as well as other flavivirus infections (Japanese encephalitis virus infection of cultured neurons [15], but also the infection of mouse brain neurons and BHK-21 cells with Japanese encephalitis virus [16-18] and other viral infections [14]).

Strain 263 exhibits a nonvirulent temperature-sensitive phenotype. It was shown that this virus lost its neuroinvasiveness due to mutations in the NS2B-NS3 proteolytic complex [7]. In our study, TEM images showed numerous virus-like particles in macrophage cells infected with strain 263 , but no viral replication was observed in the growth kinetics study.

In the case of virus-infected cells, the induction of early cell death would severely limit virus production and reduce or eliminate spread of progeny virus in the host. Thus, most animal viruses have evolved strategies to evade or delay early apoptosis to allow the production of high yield progeny virus [19]. In the case of the macrophage infection with the Hypr strain, necrotic macrophages were observed more frequently than apoptotic ones. This is in contrast to Hypr infection in glioblastoma and medulloblastoma cell lines [13], but in agreement with the situation observed in TBEV-infected neuroblastoma cells. In human monocytes infected with dengue virus, predominant apoptotic features were demonstrated [20].

Cells of eukaryotic organisms contain microtubules with various functions during the different developmental stages [21]. $\alpha$-Tubulin is included in a complex polymer of microtubules, while centrosomes nucleate the as- sembly and establish the polarity of microtubules. $\gamma$-Tubulin has an essential role in centrosome microtubule nucleation [22-24]. It is localized in the centrosome and in the cytoplasm centrosomes [22-26]. This suggests that the alteration of microtubule nucleation by centrosomes might not occur in mouse macrophages when infected with the Hypr strain. Nevertheless, the molecular mechanisms underlying this process will need more biochemical studies. Assessment of the immunofluorescence staining data reported here suggests that macrophage cells infected with Hypr show abnormalities of the cytoskeletal component such as the rearrangement of microtubules ( $\alpha$-tubulin). This would impair cell division, interphase cytoplasmic organization as well as other cytoskeletal functions.

The molecular and biochemical characterization of mouse macrophage resistance/susceptibility to the TBEV infection as well as the study of the interaction of the Hypr strain with human monocytes need further investigation.

\section{Acknowledgements}

We thank Dr. John R. Milne from the Department of Biology, Faculty of Science, Mahidol University, Bangkok, Thailand, for language editing. The authors acknowledge the financial support by the grants Z60220518, MSM 6007665801 of the Ministry of Education, Youth and Sports of the Czech Republic, the grant 524/06/1479 from the Grant Agency of the Czech Republic, and Research Centre of the Ministry of Education, Youth and Sports of the Czech Republic (LC 06009).

\section{References}

1 Kopecký J, Křivanec K, Tomková E: Attenuated temperature-sensitive mutants of tickborne encephalitis (TBE) virus isolated from a natural focus; in Dusbábek F, Bukva V (eds): Modern Acarol. Prague, Academia, 1991, pp 11-19.

-2 Senigl F, Kopecký J, Grubhoffer L: Distribution of $\mathrm{E}$ and NS1 proteins of TBE virus in mammalian and tick cells. Folia Microbiol (Praha) 2004;49:213-216.

-3 Khozinsky VV, Semenov BF, Gresíková M, Chunikhin SP, Sekeyová M, Kozuch O: Role of macrophages in the pathogenesis of experimental tick-borne encephalitis in mice. Acta Virol 1985;29:194-202.
-4 Kopecký J, Grubhoffer L, Tomková E: Interaction of tick/borne encephalitis virus with mouse peritoneal macrophages. The effect of antiviral antibody and lectin. Acta Virol 1991;35:218-225.

5 Pronin AV, Ozherelkov SV, Narovlyansky AN, Danilov LL, Maltsev SD, Deyeva AV, Grigorieva EA, Sanin AV: Role of cytokines in immunomodulatory effects of polyprenyl phosphate: new generation of antiviral drugs. Russ J Immunol 2000;5:155-164.

-6 Kreil TR, Burger I, Bachmann M, Fraiss S, Eibl MM: Antibodies protect mice against challenge with tick-borne encephalitis virus (TBEV)-infected macrophages. Clin Exp Immunol 1997;110:358-361.
7 Pospíšil L, Jandásek L, Pešek J: Isolation of new strains of tick-borne encephalitis virus, Brno region, summer 1953 (in Czech). Lékařské Listy 1954;9:3-5.

$>8$ Růžek D, Gritsun TS, Forrester NL, Gould EA, Golovchenko M, Rudenko M, Kopecký J, Grubhoffer L: Mutations in the NS2B and NS3 genes affect mouse neuroinvasiveness of a Western European field-strain of tickborne encephalitis virus. Virology 2008; 374 : 249-255.

9 Mauel J, Defendi V: Infection and transformation of mouse peritoneal macrophages by simian virus 40. J Exp Med 1971;134:335350. 
-10 De Madrid AT, Porterfield JS: A simple microculture method for the study of group B arboviruses. Bull WHO 1969;40:113-121.

-11 Novoa RR, Calderita G, Arranz R, Fontana J, Granzow H, Risco C: Virus factories: associations of cell organelles for viral replication and morphogenesis. Biol Cell 2005;97:147172.

12 Mackenzie JM, Westaway EG: Assembly and maturation of the flavivirus Kunjin virus appear to occur in the rough endoplasmic reticulum and along the secretory pathway, respectively. J Virol 2001;75:10787-10799.

13 Růžek D, Vancová M, Tesařová M, Ahantarig A, Kopecký J, Grubhoffer L: Morphological changes in human neural cells following tick-borne encephalitis virus infection. J Gen Virol 2009;90:1649-1658.

$\checkmark 14$ Westaway EG, Mackenzie JM, Kenney MT, Jones MK, Khromykh AA: Ultrastructure of Kunjin virus-infected cells: colocalization of NS1 and NS3 with double-stranded RNA, and of NS2B with NS3, in virus-induced membrane structure. J Virol 1997;71:66506661.
15 Wang JJ, Liao CL, Chiou YW, Chiou CT, Huang YL, Chen LK: Ultrastructure and localization of $\mathrm{E}$ proteins in cultured neuron cells infected with Japanese encephalitis virus. Virology 1997;238:30-39.

16 Hase T: Virus-neuron interactions in the mouse brain infected with Japanese encephalitis virus. Virchows Arch B Cell Pathol 1993;64:161-170.

17 Hase T, Dubois DR, Summers PL, Downs $\mathrm{MB}$, Ussery MA: Comparison of replication rates and pathogenicities between the SA 14 parent and SA14-14-2 vaccine strains of Japanese encephalitis virus in mouse brain neurons. Arch Virol 1993;130:131-143.

18 Takegami T, Sakamuro D, Furukawa T: Japa nese encephalitis virus nonstucture protein NS3 has RNA binding and ATPase activities. Virus Genes 1995;9:105-112.

19 Teodoro JG, Branton PE: Regulation of apoptosis by viral gene products. J Virol 1997;71: 1739-1746.

20 Mosquera JA, Hernandez JP, Valero N, Espina LM, Anez GJ: Ultrastructural studies on dengue virus type 2 infection of cultured humanmonocytes. VirolJDOI:10.1186/1743 422X-2-26.
21 Lajoie-Mazenc I, Détraves C, Rotaru V, Garès M, Tollon Y, Jean C, Julian M, Wright M, Raynaud-Messina B: A single gamma-tubulin gene and mRNA, but two gamma-tubulin polypeptides differing by their binding to the spindle pole organizing centres. J Cell Sci 1996;109:2483-2492.

-22 Oakley BR, Oakley CE, Yoon Y, Jung MK: Gamma-tubulin is a component of the spindle pole body that is essential for microtubule function in Aspergillus nidulans. Cell 1990;6:1289-1301.

23 Stearns T, Evans L, Kirschner M: Gammatubulin is a highly conserved component of the centrosome. Cell 1991;65:825-836.

24 Zheng Y, Jung MK, Oakley BR: Gamma-tubulin is present in Drosophila melanogaster and Homo sapiens and is associated with the centrosome. Cell 1991;65:817-823.

25 Oakley BR: Gamma-tubulin: the microtubule organizer? Trends Cell Biol 1992;2:1-5.

26 Oakley CE, Oakley BR: Identification of gamma-tubulin, a new member of the tubulin superfamily encoded by mipA gene of Aspergillus nidulans. Nature 1989;338:662664 